percritical Fields, edited by I. A. Sellin (Springer, Berlin, 1978), Vol. 5, p. 3.

${ }^{2}$ See, for example, H. W. Kugel and D. E. Murnick, Rep. Prog. Phys. 40, 297 (1977), and references cited therein.

${ }^{3}$ G. W. Erickson, Phys. Rev. Lett。27, 780 (1971), and J. Phys. Chem. Ref. Data 6, 831 (1977)。

${ }^{4}$ P. J. Mohr, Phys. Rev. Lett. 34, 1050 (1975), and Ann. Phys. (N.Y.) 88, 26 (1974), and in Beam-Foil Spectroscopy, edited by I. A. Sellin and D. J. Pegg (Plenum, New York, 1976), p. 89.

${ }^{5}$ W. R. Johnson, Phys. Rev. Lett. 29, 1123 (1972).
${ }^{6} \mathrm{M}$. Hillery and $\mathrm{P}$ 。J. Mohr, private communication; C. Y。 Fan, M. Garcia-Munoz, and I. A. Sellin, Phys. Rev。161, 6 (1967).

7J. Alonso, D. Dietrich, H. Gould, and R. Marrus, private communication.

${ }^{8}$ V. F. Weisskopf, Phys. Today 13 , No.9, 24 (1960); J. Terrell, Phys. Rev. 116, 1041 (1959).

${ }^{9} \mathrm{H}$. A。 Bethe and E. E. Salpeter, Quantum Mechanics of One-and Two-Electron Atoms (Springer, Berlin, 1957), Sect. 63 .

${ }^{10} \mathrm{R}$. Marrus and R。Schmieder, Phys。Rev. A $\underline{5}, 1160$ (1972).

\title{
Doppler-Tuned Hyperfine Spectroscopy of the Lithium Ion
}

\author{
B. Fan, Allen Lurio, ${ }^{(a)}$ and D. Grischkow sky \\ IBM Thomas J. Watson Reserach Center, Yorktown Heights, New York 10598
}

(Received 23 August 1978)

\begin{abstract}
We have used the Doppler-tuned ion-beam, laser-spectroscopy method to measure the hyperfine intervals in the $2^{3} \mathrm{~S}$ and $2^{3} \mathrm{P}^{\circ}$ states of ${ }^{7} \mathrm{Li}^{+}$. Our resolution was 10 times that of the best previous measurement. In contrast to the earlier experimental work we find excellent agreement between our measurements and the theoretical values.
\end{abstract}

The fine and hyperfine structure of the $n p^{3} P^{\circ}$ and $n s^{3} S$ states of the helium isoelectronic series are of considerable interest in atomic physics because their relative simplicity allows precise theoretical calculations. The fine structure of the $n^{3} P^{\circ}$ states $(n=2-5)$ has been calculated up to $Z=10$ by Schiff and co-workers. ${ }^{1}$ Their calculations included the contributions from the masspolarization correction, relativistic effects of order $\alpha^{2}$, and mixing of the singlet and triplet states, but not the Lamb-shift correction. For $\mathrm{Li}^{+}$, the predominance of the spin-spin magnetic interactions causes the three fine-structure levels of the $2^{3} P^{\circ}$ state to be partially inverted, so that a precision measurement makes possible a different test for the correctness of the two-electron, spin-spin Hamiltonian compared to helium where spin-spin and spin-orbit terms contribute about equally. ${ }^{2}$ Jette, Lee, and Das $^{3}$ have used the linked-cluster many-body perturbation method to calculate the contributions of the magnetic contact, dipolar, and orbital hyperfine interactions to the hyperfine structure of the $2^{3} P^{\circ}$ state of lithium ions. Their results agree to about 1 part in $10^{4}$ with the more recent numerical calculations by Aahamar and Hambro. ${ }^{4}$ Questions have been raised by the recent optical $1^{506}$ and beam-foil ${ }^{7}$ experimental results, deduced from incompletely resolved hyperfine structures for $\mathrm{Li}^{+}$, which showed significant discrepancies with the accurate many-body calculations. ${ }^{8}$ Thus, it is important to make our independent high-resolution measurements.

It was first demonstrated by Wing et al. ${ }^{9}$ that the velocity-bunching effect, where the energy spread remains the same but the velocity spread is reduced with higher energies for accelerated ions, can be utilized in high-resolution laser spectroscopy. Linewidths can be significantly reduced, and the signal from the low-density, fast ion beam can thereby be increased by efficient collinear excitation with monochromatic laser light. This technique has been used in the spectroscopy of molecular $\mathrm{HD}^{+}$ions, ${ }^{9} \mathrm{Ba}^{+}$ions, ${ }^{10}$ $\mathrm{Xe}^{+}$ions, ${ }^{11}$ and neutral $\mathrm{Na}$ and $\mathrm{Cs}$ atoms ${ }^{12}$

We have used this ion-beam, laser-spectroscopy method to measure the hyperfine intervals of the metastable $2^{3} S$ and the $2^{3} P^{\circ}$ states depicted in Fig. 1 for the lithium ion ${ }^{7} \mathrm{Li}^{+}$. In Fig. 2, our observed linewidth is seen to be $\frac{1}{10}$ that of the most accurate previous optical measurement, ${ }^{6}$ where the linewidth was due to Doppler broadening from a liquid-nitrogen-cooled hollow-cathode lamp. Our narrow linewidth allowed us to resolve completely all the hyperfine transitions and to thereby obtain the hyperfine intervals with much improved accuracy. Our results, presented in Table I, show that in contrast to the previous work, we obtain excellent agreement with the theory of Jette, Lee, and Das ${ }^{2}$ for all the 


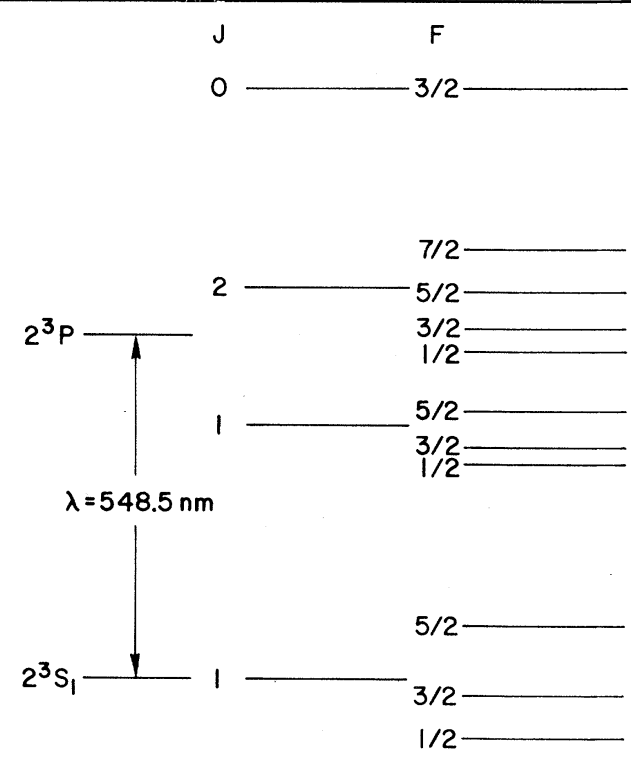

FIG. 1. Schematic energy level diagram showing the fine and hyperfine structure of the $2^{3} S_{1}$ and $2^{3} P^{\circ}$ levels of the ${ }^{7} \mathrm{Li}^{+}$ion. The metastable $2^{3} S_{1}$ state is $59 \mathrm{eV}$ above the $1^{1} S_{0}$ singlet ground state of ${ }^{7} \mathrm{Li}^{+}$and has a lifetime of $49 \mathrm{sec}$.

\section{hyperfine intervals.}

The $\mathrm{Li}^{+}$ion beam is produced, accelerated, and mass analyzed in an Extrion 50-keV ion-implanation machine. The key component to the success of the experiment is the sputter-ion source which produces a $\mathrm{Li}$ ion beam which $10^{-6}$ to $10^{-7}$ of the total number of ions are in the metastable $2^{3} S_{1}$ state. A mixture of helium and argon is used as the sputtering gas and the anode target is a pellet of compressed LiF powder. On emerging from the accelerator the ion beam is electrostatically deflected by $45^{\circ}$. A small hole in one of the deflection plates permits entry of the laser beam to overlap the ion beam trajectory A single-mode, cw, Rhodamine 110 dye laser

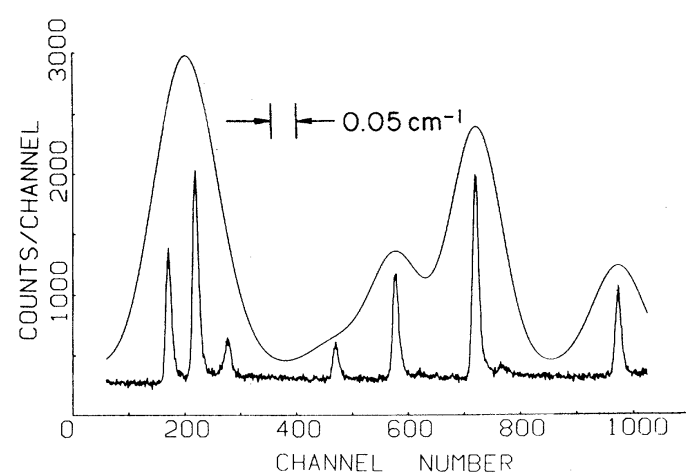

FIG.2. Experimental hyperfine spectra of the $2^{3} S_{1}$ $2^{3} P_{1}{ }^{\circ}$ transition. The upper curve shows the best previous optical measurement (Ref. 6). Our result of laser-induced fluorescence (the lower curve) demonstrates the tenfold improved resolution. The channel numbers are approximately proportional to both the post-accelerating voltage and the frequency sweep. The separation between the lowest- and highest-frequency peaks is $1.052 \mathrm{~cm}^{-1}$.

with an output power of $10 \mathrm{~mW}$ is used to excite the lithium ions. With a typical ion energy of 40 $\mathrm{keV}$, the mean ion velocity is $0.0037 c$, giving a Doppler shift of $2 \mathrm{~nm}$ for the $2^{3} S-2^{3} P^{\circ}$ hyperfine transitions. In our experiment, we use the technique introduced by Dufay et al. ${ }^{10}$; the laser frequency is fixed at $546.5 \mathrm{~nm}$, and the hyperfine transitions are finely scanned by sweeping the ion velocity. This is accomplished by applying a small accelerating or decelerating electric field to the ions before they enter the observation chamber. In this chamber the laser is in resonance with the scanned hyperfine transition, and we monitor the fluorescence from this excited transition.

The laser-induced fluorescence emitted normal to the beams is collected with a cylindrical lens $(f=4 \mathrm{~cm})$ over a flight path of $2 \mathrm{~cm}$, and is detected with a cooled photomultiplier. Because of

TABLE I. Hyperfine intervals in ${ }^{7} \mathrm{Li}^{+}$(in inverse centimeters).

\begin{tabular}{rrccccc}
\hline & & Expt. $^{\mathrm{a}}$ & Expt. $^{\mathrm{b}}$ & Expt. $^{\mathrm{c}}$ & Our work & Theory $^{\mathrm{d}}$ \\
\hline${ }^{3} S_{1}$ & $F(1 / 2-3 / 2)$ & $0.386(50)$ & $\ldots$ & $0.3944(72), 0.3968(35)$ & $0.3960(4)$ & 0.3962 \\
& $(3 / 2-5 / 2)$ & 0.643 & $\ldots$ & $0.6575(44), 0.6613(50)$ & $0.6604(8)$ & 0.6603 \\
${ }^{3} P_{1}{ }^{\circ}$ & $F(1 / 2-3 / 2)$ & 0.138 & $0.1323(13)$ & $0.1470(98)$ & $0.1409(6)$ & 0.1409 \\
& $(3 / 2-5 / 2)$ & 0.341 & $0.3156(31)$ & $0.3299(51)$ & $0.3313(8)$ & 0.3316 \\
${ }^{3} P_{2}{ }^{\circ}$ & $F(1 / 2-3 / 2)$ & 0.216 & $0.1944(19)$ & $0.1898(34)$ & $0.2062(6)$ & 0.2069 \\
& $(3 / 2-5 / 2)$ & 0.333 & $0.3087(31)$ & $0.3269(38)$ & $0.3203(4)$ & 0.3204 \\
& $(5 / 2-7 / 2)$ & 0.404 & $0.3785(38)$ & $0.3862(31)$ & $0.3923(4)$ & 0.3926 \\
\hline \hline
\end{tabular}

$\begin{array}{llll}{ }^{\mathrm{a}} \text { Ref. } 5 . & { }^{\mathrm{b}} \text { Ref. } 7 . & { }^{\mathrm{d}} \text { Ref. } 6 . & \end{array}$


the low signal level, photon counting is used. The photons collected in repeated scans of the post-acceleration voltage are accumulated in a multichannel analyzer with the channel numbers synchronized to this voltage. Because of the frequency of the fluorescent light emitted normal to the beams is not Doppler shifted, its wavelength of $548.5 \mathrm{~nm}$ differs from that of the laser by 2.0 $\mathrm{nm}$. This situation allows us to use a narrowband interference filter to reduce the laser-scattering count rate to 40 counts/sec; the dark count is 20 /sec. Even though the pressure in the observation region is low, typically $2 \times 10^{-7}$ Torr, the broad-band radiation produced by the collisions between the ion beam and residual gases results in a count rate of about 160 counts/sec for an ion beam current of $2 \mu \mathrm{A}$. The fluores cence signal is 1000 counts/sec for the strongest transitions. The linewidth of the Doppler-shifted fluorescence signal depends on the sputtering conditions in the ion source. With low gas pressure in the source, we routinely observe a width of $450 \mathrm{MHz}$ (full width at half maximum) compared to the 8-MHz natural width. The laser frequency jitter contributes about $60 \mathrm{MHz}$.

An example of our experimental results is the complete hyperfine spectrum shown in Fig. 2. The transitions are labeled in terms of the $F$ values (indicated in Fig. 1) for the $2^{3} S_{1}$ and $2^{3} P_{1}{ }^{\circ}$ states, respectively, and in order of increasing channel number as $\left(\frac{1}{2}, \frac{3}{2}\right),\left(\frac{3}{2}, \frac{5}{2}\right),\left(\frac{1}{2}, \frac{1}{2}\right),\left(\frac{3}{2}, \frac{3}{2}\right),\left(\frac{3}{2}\right.$, $\left.\frac{1}{2}\right),\left(\frac{5}{2}, \frac{5}{2}\right)$, and $\left(\frac{5}{2}, \frac{3}{2}\right)$. From an inspection of this list it is clear the the hyperfine intervals of the $2^{3} S_{1}$ and $2^{3} P_{1}^{\circ}$ states (tabulated in Table I) can each be twice independently determined. In order ot measure the frequency separation between any two lines, the line centers and the total corresponding accelerating voltages must be obtained. The total voltage is the sum of the source voltage, main accelerating voltage, and post-acceleration voltage. The total voltage is known to within $0.05 \%$ and the principal uncertainty is due to the fluctuations $( \pm 10 \mathrm{~V})$ in the source voltage. The main accelerating voltage is known to within a few volts. The post-acceleration voltage has been calibrated versus channel number with better than $10^{-4}$ accurarcy. Once these two total voltages are known, the corresponding velocities and their difference can be calculated and the frequency difference obtained. For our data in Table I, we use a finer frequency scale (keeping the same number of channels) and only scan through a portion of the hyperfine structure. This permits the line centers to be determined much more accurately, and we obtain the hyperfine intervals with a typical accuracy of \pm 0.0005 $\mathrm{cm}^{-1}$. Systematic errors are minimized by checking the self-consistency within the same fluorescence spectrum and taking spectra at different ion energies.

Our results are compared with the previous works (ordered chronologically) in Table I. The first relatively accurate measurements of the hyperfine structure were obtained by Herzberg and Moore ${ }^{5}$ using a 21-ft grating spectrograph. The measurements stated a conservative figure of $0.05 \mathrm{~cm}^{-1}$ for their experimental error. They have assumed the same hyperfine splitting factors for both $2^{3} S$ and $2^{3} P^{\circ}$ states in order to interpret their blended spectra and to calculate the hyperfine intervals. For the later beam-foil results of Berry and co-workers, ${ }^{7}$ the claimed accuracy is better than $1 \%$. The large disagreement $(3-6 \%)$ with the theoretical calculations of Jette, Lee, and Das $^{3}$ can be partly attributed to the fitting analysis in Ref. 7 based on only the contact part of the spin Hamiltonian. However, as this approximation and other corrections cannot account for more than $\frac{1}{3}$ of the total disagreement (see Ref. 3), systematic errors still perhaps dominate. The previous most accurate measurements were made by Bacis and Berry, ${ }^{6}$ who used a scanning Fabry-Perot to analyze the hyperfine structures from a liquid-nitrogencooled hollow-cathode lamp. Their resolution is shown in Fig. 2, and because of the Doppler linewidth, they used a seven-parameter fit to the blended spectra. They claim an absolute calibration accuracy of $0.001 \mathrm{~cm}^{-1}$. However, as indicated in the table, differences larger than 0.001 $\mathrm{cm}^{-1}$ occur for the same hyperfine intervals of the $2^{3} S_{1}$ state, when obtained from their different $2^{3} S_{1}-2^{3} P_{J}^{\circ}$ transition frequencies. In addition there are relatively large discrepancies, ranging up to $0.0171 \mathrm{~cm}^{-1}$, between their results and the theory. ${ }^{3}$ This fact led to concern ${ }^{6.7}$ as to whether the theory was indeed accurate. However, the theoretical calculation is believed to be accurate to better than $0.0001 \mathrm{~cm}^{-1}$.

In contrast to the situation above, our results show excellent agreement with theory. The largest is $0.0007 \mathrm{~cm}^{-1}$ which is at the limit of our experimental error. It should be emphasized that our experimental hyperfine intervals are calculated directly from the completely resolved spectra and are theory independent. An additional check on our results is the ratio of the two ${ }^{3} S_{1}$ intervals. According to the Lande interval rule, 
this ratio should be 0.6000 , and our experimental value is 0.5996. Consequently, we conclude that the theory of Jette, Lee, and Das ${ }^{3}$ is accurate within the limits of our measurements.

The expert technical assistance of J. Keller and N. A. Penebre is gratefully acknowledged. We would also like to thank M. M. T. Loy, A. H. Nethercot, and J. J. Wynne for helpful discussions. This work was partially supported by the U. S. Office of Naval Research.

\section{(a) Deceased.}

${ }^{1}$ B. Schiff, Y. Accad, and C. L. Pekeris, Phys. Rev. A 1, 1837 (1970); Y. Accad. C. L. Pekeris, and B. Schiff, Phys. Rev. A $\underline{4}, 516$ (1971); B. Schiff, Y. Accad, and C. L. Pekeris, Phys. Rev. A 8, 2272 (1973).

${ }^{2}$ A. Adler, W. Kahn, R. Novick, and T. Lucatorto, Phys. Rev. A $\underline{7}, 967$ (1973).

${ }^{3}$ A. N. Jette, T. Lee, and T. P. Das, Phys. Rev. A $\underline{9}$, 2337 (1974).

${ }^{4}$ K. Aashamar and L. Hambro, J. Phys. B 10, 553 (1977).

${ }^{5}$ G. Herzberg and H. R. Moore, Can. J. Phys. $\underline{37}$,
1293 (1959).

${ }^{6}$ H. G. Berry and R. Bacis, Phys. Rev. A $\underline{8}, 36$ (1973); R. Bacis and H. G. Berry, Phys. Rev. A 10, 466 (1974).

${ }^{7}$ H. G. Berry and J. L. Subtil, Phys. Rev. Lett. 27, 1103 (1971); H. G. Berry, J. L. Subtil, E. H. Pinnington, H. J. Andra, W. Wittmann, and A. Gaupp, Phys. Rev. A 7, 1609 (1973); H. G. Berry, E. H. Pinnington, and J. L. Subtil, Phys. Rev. A 10, 1065 (1974).

${ }^{8} \mathrm{~A}$ preliminary, unpublished measurement of the hyperfine interval between the $2^{3} P_{1}^{0}, F=2 \hookrightarrow F=1$ states of ${ }^{6} \mathrm{Li}^{+}$was made by A. Adler, T. Lucatorto, R. Novick, and G. F. A. Sprott [see Columbia Radiation Laboratory (Columbia University) Progress Report No. 19, August 1969 (unpublished)]. This experiment is also described in Ref. 2.

${ }^{9}$ W. H. Wing, G. A. Ruff, W. E. Lamb, Jr., and J. Spezeski, Phys. Rev. Lett. 36, 1488 (1976).

${ }^{10}$ M. Dufay, M. Carre, M. L. Gaillard, G. Meunier, H. Winter, and A. Zganski, Phys. Rev. Lett. 37, 1678 (1976); H. Winter and M. L. Gaillard, Z. Phys. A281, 311 (1977), and J. Phys. B 10, 2739 (1977).

${ }^{11} \mathrm{~T}$. Meier, H. Huhnermann, and H. Wagner, Opt. Commun. 20, 397 (1977).

${ }^{12}$ K.-R. Anton, S. L. Kaufman, W. Klempt, G. Moruzzi, R. Neugart, E.-W. Otten, and B. Schinzler, Phys. Rev. Lett. $\underline{40}, 642$ (1978).

\title{
Observation of Electric-Field-Induced Resonances above the Ionization Limit in a One-Electron Atom
}

\author{
R. R. Freeman, N. P. Economou, and G. C. Bjorklund \\ Bell Laboratories, Holmdel, New Jersey 07733 \\ and \\ K. T. $\mathrm{Lu}$ \\ Argonne National Laboratory, Argonne, Illinois 60439 \\ (Received 19 April 1978)
}

\begin{abstract}
Measurements of the relative photoionization cross sections of $\mathrm{Rb}$ in the presence of various strengths of external electric fields are reported. Systematic, field-dependent, resonance structure is observed not only for energies above the classical field-ionization limit, but above the zero-field ionization limit as well. A striking dependence of the cross section upon light polarization is also observed.
\end{abstract}

We report the results of an investigation of the wavelength dependence of the single-photon photoionization cross section of ground-state $\mathrm{Rb}$ in the presence of externally applied electric fields. For each field value, a systematic resonance structure versus wavelength was observed, not only for energies above the classical field-ionization threshold (which has been previously reported), but above the zero-field ionization limit as well. The observation of electric-field-dependent resonances in the photoionization cross section above the zero-field limit in a one-electron-like atom is surprising and to our knowledge has not been previously reported. In this Letter we present some relevant results of our experiment, discuss the physical mechanism responsible, develop a model whose predictions are in good agreement with our data, and attempt to place the results in proper perspective relative to recent measurements of field ionization and Stark effects in alkali atoms.

For detailed study of the cross section, we employed a collison-free, low-density $\left(\lesssim 10^{9} / \mathrm{cm}^{3}\right)$ atomic beam. The $\mathrm{Rb}$ atomic beam was irradi- 